variability of the artery were described and then analysed for association with clinical factors using a random effects regression model. Results 298 arteries were analysed from 48 patients (mean age 60 years). The mean lateral distance from the spine over which the artery was exposed within the intercostal space ("unsafe artery length') was $39 \mathrm{~mm}$ with wide variability (SD=10 $\mathrm{mm}$, 10th to 90th centile $28 \mathrm{~mm}$ to $51 \mathrm{~mm}$, Abstract P64 Figure 1A). At $3 \mathrm{~cm}$ lateral distance from the spine $16.6 \%$ of arteries were shielded by the superior rib, compared to $96.6 \%$ at $6 \mathrm{~cm}$. Unsafe artery length was not associated with age, sex, rib space or side. Using regression modelling, variability of arterial position (as SD and coefficient of variation) was significantly associated with age (coeff $0.91, \mathrm{p}<0.001)$ and rib space number (coeff $-2.60, \mathrm{p}<0.001$ ) (Abstract P64 Figure 1B). Variability of arterial position was strongly negatively correlated with lateral distance from the spine (Pearson's $-0.77, \mathrm{p}<0.001$ ).
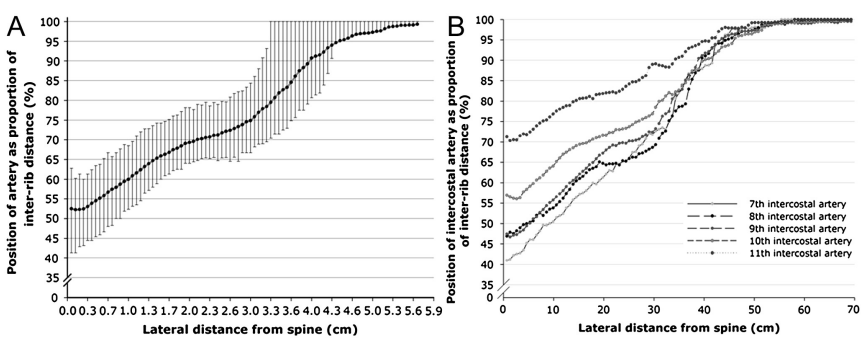

Abstract P64 Figure 1 (A). Position of artery as proportion of inter-rib distance (\%). (B). Position of intercostal artery as proportion of inter-rib distance (\%).

Conclusions The intercostal artery is exposed within the intercostal space in the first $6 \mathrm{~cm}$ lateral to the spine; variability of its vertical position is greater in older patients and more cephalad rib spaces. This implies that pleural interventions within $6 \mathrm{~cm}$ lateral to the spine should be conducted with caution and that the risk of intercostal artery laceration is potentially higher in older patients and more cephalad rib spaces.

\section{P65 IS SERUM N-TERMINAL PRO B TYPE NATRIURETIC PEPTIDE (NT-PROBNP) MEASUREMENT USEFUL IN THE INVESTIGATION OF UNILATERAL PLEURAL EFFUSIONS?-A PROSPECTIVE OBSERVATIONAL STUDY}

doi:10.1136/thx.2010.150979.16

${ }^{1} \mathrm{C}$ E Hooper, ${ }^{2} \mathrm{l}$ Rider, ${ }^{3} \mathrm{R}$ S Finn, ${ }^{4} \mathrm{~A} J \mathrm{~J}$ Morley, ${ }^{3} \mathrm{~J}$ E Harvey, ${ }^{2} \mathrm{~A}$ Skyrme-Jones, ${ }^{1} \mathrm{~N}$ A Maskell. ${ }^{1}$ Academic Respiratory Unit, Department of Clinical sciences, University of Bristol, Southmead Hospital, Bristol, UK; ${ }^{2}$ Southmead Hospital, North Bristol NHS Trust, Bristol, UK; ${ }^{3}$ North Bristol Lung Centre, Southmead Hospital, Bristol, UK; ${ }^{4}$ Pleural Clinical Trials Unit, Southmead Hospital, Bristol, UK

Measurement of serum NT-proBNP has been proposed in the investigation of pleural effusions, particularly in the diagnosis of cardiac failure in those misclassified as exudates by Light's criteria. Studies have reported excellent diagnostic accuracy for the test but have included both bilateral and unilateral effusions and applied short follow-up periods. We prospectively examined the diagnostic utility of serum NT-proBNP in a consecutive series of unilateral pleural effusions with robust follow-up and diagnostic criteria.

Method Consecutive patients presenting to a UK teaching hospital with an undiagnosed unilateral pleural effusion underwent clinical assessment including CXR, ECG, echocardiogram, thoracentesis (and CT when appropriate). Light's criteria were applied. Serum NTproBNP was measured using point of care ELISA. Patients were followed up to histological/microbiological diagnosis, radiographic resolution or 12 months. Echocardiograms were double reported and diagnosis determined independently by two respiratory consultants-all blind to NT-proBNP results.

Results 118 patients. Median age 74 (42-95). 39 in patients, 79 outpatients. 18 transudates, 92 exudates. 30 large, 66 moderate, 22 small. Diagnoses: primary cardiac cause (PCC) 20/118, Malignant $57 / 118$, PE $4 / 118$, Non-cardiac transudate $3 / 118$, other benign cause 30/118. The ROC curve for NT-proBNP discriminating effusions of PCC gave an AUC of 0.845 (0.774-0.934). At cut-off of age and sex adjusted 97.5 th centile (healthy population) NT-proBNP had sensitivity $100 \%$, Specificity 53\%, PPV 30\% and NPV 100\% and all four cardiac exudates were correctly diagnosed. At an optimum cutoff of $1500 \mathrm{pg} / \mathrm{ml}$-sensitivity $75 \%$, specificity $76 \%$, PPV $38 \%$ and NPV 94\%. Co-morbid cardiac disease was common in patients without a PCC for effusion with $70 \%$ having significant abnormalities on echocardiogram but cardiac disease was considered to be contributing to effusion in only $9 / 98$ of this group.

Conclusion The excellent negative predictive value of NT-proBNP, particularly at an age and sex adjusted cut-off level gives the test utility to rule out a primarily cardiac cause in selected cases of unilateral pleural effusion. Co-morbid cardiac disease and associated NT-proBNP elevation is very common in patients with a noncardiac origin of pleural effusion such that a positive test at baseline should not alter the initial investigation pathway, particularly amongst pleural exudates.

\section{P66 HISTONE DEACETYLASE INHIBITOR CBHA ATTENUATES THE EXPRESSION OF PLASMINOGEN ACTIVATOR INHIBITOR-1 IN HUMAN PLEURAL MESOTHELIAL CELLS}

doi:10.1136/thx.2010.150979.17

${ }^{1}$ Chi-Li Chung, ${ }^{2}$ Wei-Lin Chen, ${ }^{3} Y u-W e n$ Cheng, ${ }^{2}$ Yung-Chen Chou, ${ }^{2}$ Ming-Jen Hsu, ${ }^{1}$ Che-Jen Hsiao, ${ }^{2}$ Joen-Rong Sheu, ${ }^{2}$ George Hsiao. ${ }^{1}$ Department of Chest Medicine, Taipei Medical University Hospital and School of Respiratory Therapy, College of Medicine, Taipei Medical University, Taipei, Taiwan; ${ }^{2}$ Graduate Institute of Medical Sciences and Department of Pharmacology, College of Medicine, Taipei Medical University, Taipei, Taiwan; ${ }^{3}$ Department of Pharmacy, School of Pharmacy, Taipei Medical University, Taipei, Taiwan

Background Plasminogen activator inhibitor-1 (PAI-1), primarily upregulated by transforming growth factor (TGF)- $\beta$, is essential for development of fibrosis. Histone deacetylases (HDACs) have been shown to modulate gene expression and fibrogenesis in various tissues. However, the implications of HDAC in PAI-1 expression and pleural fibrosis remain unclear. We examined the effects of $m$-carboxycinnamic acid bis-hydroxamide (CBHA), a hybrid-polar HDAC inhibitor, on PAI-1 expression in a human pleural mesothelial cell line (MeT-5A). Methods MeT-5A cells were treated with TGF- $\beta 1(10 \mathrm{ng} / \mathrm{ml})$ in the presence or absence of CBHA $(0.2-1 \mu \mathrm{M})$. The expression and stability of PAI-1 mRNA and protein, PAI-1 promoter activity, activation of Smad signaling, and protein-protein interactions of Smads with transcriptional cofactors Sp1 and coactivator p300 were assayed using the methods of Western blotting, reverse transcription-polymerase chain reaction, transient transfection and luciferase activity assay, immunoflurescence staining and immunoprecipitation, respectively.

Results CBHA significantly inhibited TGF- $\beta 1$-induced PAI- 1 mRNA and protein expression, and attenuated PAI-1 promoter activity in MeT-5A cells. CBHA abrogated TGF- $\beta 1$-induced Smad4 nuclear translocation, but not Smad2/3 activation. Furthermore, the TGF$\beta 1$-induced association of Smad4 with p300, but not with Sp1, was disrupted by CBHA. Alternatively, CBHA accelerated PAI-1 mRNA degradation, possibly through suppression of the mRNA stabilizing protein nucleolin (Abstract P66 Figure 1). 


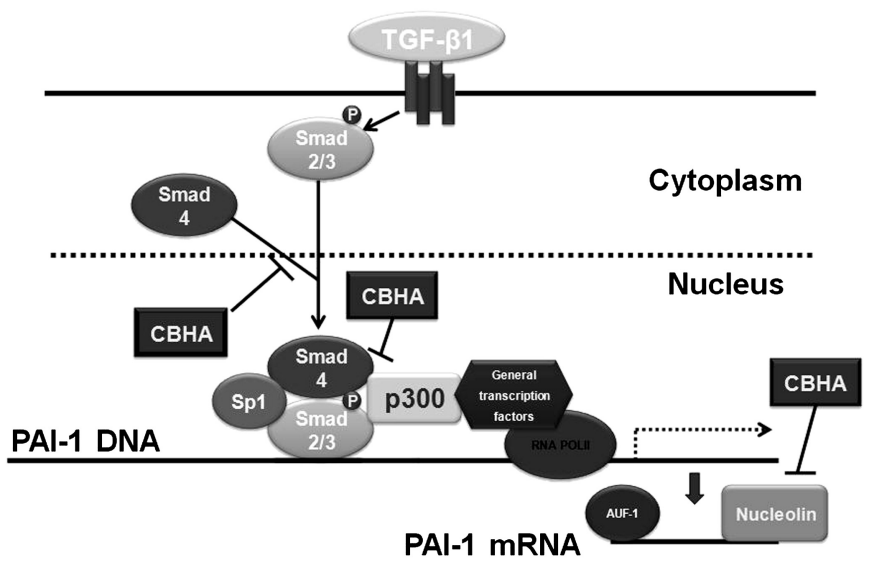

Abstract P66 Figure 1

Conclusion Our data suggests that inhibition of HDAC activity by CBHA may attenuate TGF- $\beta 1$-induced PAI- 1 expression in human pleural mesothelial cells through modulation of cellular signaling at multiple levels. HDAC inhibitors may be employed as potential therapeutic agents for pleural fibrosis.

\section{P67 OUTCOMES FROM SURGICAL MANAGEMENT OF PLEURAL INFECTION: 12-YEAR EXPERIENCE FROM A TERTIARY CARDIOTHORACIC CENTRE}

doi:10.1136/thx.2010.150979.18

${ }^{1} \mathrm{D} J \mathrm{~B}$ Marks, ${ }^{2} \mathrm{M}$ Fisk, ${ }^{1} \mathrm{C}$ Y Koo, ${ }^{2} \mathrm{~S} F$ Lee, ${ }^{2} \mathrm{D}$ Lawrence, ${ }^{1} \mathrm{R} \mathrm{F}$ Miller, ${ }^{1} \mathrm{~A}$ Zumla. ${ }^{1}$ University College London, London, UK; ${ }^{2}$ Heart Hospital, London, UK

Introduction and Objectives Thoracic empyema affects $>65000$ patients/year in the US and UK. Up to $50 \%$ require surgical drainage, and $15 \%$ die. We describe clinical features, microbiology, risk factors and surgical outcomes from referrals to a regional specialist cardiothoracic centre over 12 year.

Methods Patients were identified by searching the hospital Clinical Data Repository for a diagnosis of 'pyothorax' from 1999 to 2010. A retrospective observational study was conducted using case note and database review.

Results 406 distinct empyemas were identified. Patients were predominantly male ( $\mathrm{n}=301,74.1 \%$ ), with median age of 53 years $(\mathrm{IOR}=37-69)$. Pyothorax predominantly developed secondary to community-acquired pneumonia, although 51 (12.6\%) were hospitalacquired; $70(17.2 \%)$ were receiving steroids or immunosuppressants, $35(8.6 \%)$ had concurrent malignancy, 33 (8.1\%) were diabetic and 14 (3.4\%) patients were HIV-infected. Empyemas were right-sided in 233 $(57.3 \%, p=0.03)$; four were bilateral. A causative organism was identified in $229(56.4 \%)$ patients. Positive cultures were obtained from sputum in $59(14.5 \%)$, pleural fluid in $174(42.9 \%)$ and blood in 61 $(15.0 \%)$ patients. Organisms identified included Streptococcus milleri $(\mathrm{n}=18,4.4 \%)$, Streptococcus pneumoniae $(\mathrm{n}=39,9.6 \%)$, other streptococci $(n=10,2.5 \%)$, Enterobacteraciae $(n=12,3.0 \%)$, anaerobes $(n=32$, 7.9\%), methicillin-sensitive Staphylococcus aureus ( $\mathrm{n}=36,8.9 \%$ ), methicillin-resistant Staphylococcus aureus $(\mathrm{n}=25,6.2 \%)$, Enterococcus $(\mathrm{n}=14,3.4 \%)$, Mycobacterium tuberculosis ( $\mathrm{n}=36,8.9 \%$ ), non-tuberculous mycobacteria $(n=1,0.2 \%)$, other bacteria $(n=25,6.2 \%)$, and fungi ( $n=9,2.2 \%) ; 34(8.4 \%)$ cultures were polymicrobial. $277(68.2 \%)$ patients were managed by open thoracotomy. 116 (28.6\%) underwent video-assisted thoracoscopic surgery (VATS), of whom 17 (14.7\%) were converted to open. Three $(0.7 \%)$ required a Clagett window; 10 $(2.5 \%)$ were managed with tube thoracostomy alone. A significant trend towards increased use of VATS over time was noted $(\mathrm{p}=0.0002)$. All-cause complication rate was $20.7 \%$, and 28 -day mortality was $5.7 \%$. Low preoperative haemoglobin was predictive of mortality $(p=0.04)$, but admission and peak C-reactive protein were not.
Conclusions In this large series of empyemas from a tertiary referral centre, microbiological diagnosis was achieved in only half the patients. The identification of tuberculosis in 36 cases means routine screening for mycobacteria is necessary. Use of VATS has increased significantly, and mortality was lower than previously reported and associated with preoperative anaemia.

Contributors DJBM and MF contributed equally to this work.

\section{P68 SURGERY FOR MESOTHELIOMA: THE CASE FOR MACROSCOPIC COMPLETE RESECTION}

doi:10.1136/thx.2010.150979.19

K K W Lau, A Nakas, D A Waller. Glenfield Hospital, Leicester, UK

Objective The role for radical surgery for Malignant Pleural Mesothelioma (MPM) remains controversial. There are advocates of less radical 'debulking surgery' who argue against the need for removal of diaphragm and pericardium because of increased morbidity. We test the hypothesis that survival is increased by the more radical operations intended to remove all visible tumour and achieve macroscopic complete resection (MCR).

Methods Over a 13-year period, 362 patients underwent therapeutic surgery for MPM: in-group MCR: 232 (64\%) patients underwent either extra-pleural pneumonectomy (135 patients $(37 \%)$ ) or radical pleurectomy-decortication (97 patients $(27 \%)$ ) with resection of diaphragm and pericardium. Whilst in group D: 130 (36\%) underwent debulking surgery leaving macroscopic tumour in situ (55 patients $(15 \%)$ by thoracotomy and 75 patients $(21 \%)$ by VATS). The patients in the MCR group were younger (mean age 57.9 vs $66.1, \mathrm{p}<0.0001)$ and more had epithelioid subtype (167 (72\%) vs 86 $(66.2 \%), p<0.0001)$. We compared the hospital outcomes and overall survival between these two groups.

Results The mean length of stay was longer in group MCR than in group D (16.0 days vs 9.6 days, $\mathrm{p}<0.0001)$ but 30 -day mortality was similar $(12(5.2 \%)$ vs $8(6.2 \%), p=0.811)$. In univariate analysis, overall survival was significantly longer in the MCR group (mean survival 22 months vs 13 months, $\mathrm{p}<0.0001$ ). A similar benefit was observed for the epithelioid pathological subgroup (mean survival 25.8 months vs 16.6 months, $p<0.0001)$. Cox regression showed that surgery intended to achieve MCR was associated with a significantly reduced hazard of death after adjusting for age and pathological subtype (HR $0.72,95 \%$ CI 0.55 to $0.93, p=0.014$ ).

Conclusions Surgery to increase survival in mesothelioma should be based on an intent to achieve macroscopic complete resection. This strategy should form the basis of future trials to evaluate the role of surgery in this disease.

\section{P69 MESOTHELIOMA IN THE 21ST CENTURY. A 1-YEAR SURVEY OF MALIGNANT MESOTHELIOMA IN THE SOUTH WEST OF THE UK}

doi:10.1136/thx.2010.150979.20

R Bhatnagar, S Earl, K Lansdell, T J Howell. Derriford Hospital, Plymouth, UK

Introduction Malignant mesothelioma $(\mathrm{MM})$ is a cancer that most commonly arises within the pleura, and carries a poor prognosis. There is a strong association with past asbestos exposure so incidence varies across the UK. In the South West of the UK there is a high incidence with many cases being due to asbestos exposure in the ship-building industry.

Aims To describe the demographics, treatment strategies and survival of patients presenting with MM across the Peninsula cancer network, a population of around 1.7 million people, over a 1-year period.

Method Cases of MM diagnosed in 2007 were identified retrospectively and reviewed following interrogation of cancer, MDT, 